\title{
The Fermi scale as a focus point of high-scale gauge mediation
}

\section{F. Brümmer and W. Buchmüller}

Deutsches Elektronen-Synchrotron DESY, Notkestraße 85, D-22607 Hamburg, Germany

E-mail: felix.bruemmer@desy.de, buchmuwi@mail.desy.de

ABSTRACT: We consider the minimal supersymmetric Standard Model with large scalar and gaugino mass terms at the GUT scale, which are generated predominantly by gaugemediated supersymmetry breaking. For certain ratios of GUT-scale masses, determined by the messenger indices, large radiative corrections lead to a small electroweak scale in a way which resembles the well-known focus point mechanism. The Fermi scale, the gravitino mass and the higgsino masses are of comparable size. For a Higgs mass of about $124 \mathrm{GeV}$ all other superparticles have masses outside the reach of the LHC.

KEYwORDS: Supersymmetric Standard Model, GUT

ARXIV EPRINT: 1201.4338 


\section{Contents}

1 Introduction 1

2 Framework 2

3 A focus point from high-scale gauge mediation 3

$\begin{array}{lll}4 & \text { Phenomenology } & 7\end{array}$

$\begin{array}{llr}5 & \text { Fine-tuning } & 8\end{array}$

6 Conclusions 10

\section{Introduction}

The minimal supersymmetric Standard Model (MSSM) is increasingly coming under pressure from the results of LHC searches. The non-observation of squarks and gluinos has defied expectations to find them just above the pre-LHC exclusion bounds. In most generic scenarios they must now be heavier than about a TeV. Perhaps more severely, evidence for a Higgs boson from both ATLAS [1] and CMS [2] points to a Higgs mass of around $124-126 \mathrm{GeV}$. Should this evidence solidify, this would pose a serious naturalness problem for the MSSM, or at least for many top-down scenarios which assume a "great desert" between the TeV scale and the GUT scale. The reason is that such a large Higgs mass must be due to large loop corrections involving multi-TeV third-generation squarks, or near-maximal squark mixing. On the other hand, the third-generation squark mass and gaugino mass contributions dominate the renormalization group (RG) evolution of the Higgs soft masses. The natural size of the parameters governing the Higgs potential is therefore of the order of these large soft masses. If the electroweak symmetry breaking (EWSB) scale is to come out smaller than the soft mass scale by an order of magnitude or more, this requires large cancellations, and a corresponding fine-tuning of the GUT-scale boundary conditions.

It is conceivable that a "little hierarchy" of this kind is a consequence of certain relations among the GUT-scale soft terms, or that such relations at least reduce the degree of fine-tuning. The best-known example is perhaps focus point supersymmetry [3-7], which rests on the observation that, for comparatively small gaugino masses and at least moderately large $\tan \beta$, the scalar soft mass contributions to the EWSB order parameter $m_{Z}^{2}$ cancel through RG running. For this to happen, all scalar soft masses should be given by some universal $m_{0}$ at the GUT scale. The cancellation is then quite insensitive to the actual value of $m_{0}$ (although, of course, it only holds at a finite level of precision). Therefore, it has been argued that increasing $m_{0}$ to several $\mathrm{TeV}$ does not render the model any more unnatural. 
As the main source of fine-tuning is often the gluino mass rather than the scalar soft masses, there have also been searches for similarly favourable relations between gaugino masses in models without gaugino mass unification [8,9] (see also [10] for a related recent study).

This note serves to point out that focus point-like models may be constructed in the framework of high-scale gauge mediation with split messenger multiplets, as proposed in [11]. In these models, soft gaugino and scalar masses receive large gauge-mediated contributions. Gauge-mediated soft terms are dictated by discrete parameters, namely the messenger indices. For certain models the messenger indices are such that the contributions to $m_{Z}^{2}$ cancel between the various soft terms during $R G$ running. The soft terms also receive subdominant contributions from gravity mediation. Since the large gauge-mediated contributions cancel, the EWSB scale is naturally set by the subdominant gravity-mediated terms, and these can well be of the order of $m_{Z}^{2}$ without conflicting experiment. Our model thus shows that it is still possible to reconcile the MSSM with the latest LHC results within a well-motivated GUT framework. This can even be achieved without relying on large stop mixing, which is the mechanism underlying most bottom-up attempts to maximize the lightest Higgs mass in the MSSM, in light of the recent LHC results [12-16].

\section{Framework}

Our models are inspired by GUT-scale string compactifications, which often predict a large number of vector-like states in incomplete GUT multiplets. These exotics should decouple close to the GUT scale in order to preserve (approximate) gauge coupling unification. If they couple to the hidden sector, they will serve as messenger fields for gauge-mediated supersymmetry breaking. Messenger loops generate large soft masses for the gauginos and scalars. Other soft terms and the higgsino mass $\mu$ will be induced by Planck-suppressed operators; they will be smaller but still relevant.

For definiteness, consider a model with $N_{3}$ copies $\left(\Phi_{a_{3}}^{(3)}, \widetilde{\Phi}_{a_{3}}^{(3)}\right)$ of $(\mathbf{3}, \mathbf{1})_{0} \oplus(\overline{\mathbf{3}}, \mathbf{1})_{0}, N_{2}$ copies $\left(\Phi_{a_{2}}^{(2)}, \widetilde{\Phi}_{a_{2}}^{(2)}\right)$ of $(\mathbf{1}, \mathbf{2})_{0} \oplus(\mathbf{1}, \mathbf{2})_{0}$, and $N_{1}$ copies $\left(\Phi_{a_{1}}^{(1)}, \widetilde{\Phi}_{a_{1}}^{(1)}\right)$ of $(\mathbf{1}, \mathbf{1})_{1} \oplus(\mathbf{1}, \mathbf{1})_{-1}$ as messenger supermultiplets. ${ }^{1}$ Suppose that they couple with equal strength to a goldstino background field $X=M+\theta^{2} F$, such that the superpotential is

$$
W=\sum_{i=1}^{3} \sum_{a_{i}=1}^{N_{i}} X \Phi_{a_{i}}^{(i)} \widetilde{\Phi}_{a_{i}}^{(i)} .
$$

For $M \lesssim M_{\mathrm{GUT}}$, we define

$$
m_{\mathrm{GM}} \equiv \frac{g^{2}}{16 \pi^{2}} \frac{F}{M}
$$

to be the typical gauge-mediated contribution to the soft masses per messenger pair, where $g$ is the unified gauge coupling. The one-loop gauge-mediated gaugino masses at the

\footnotetext{
${ }^{1}$ Compare for example the model in section 3.1 of [11], which effectively has $N_{1}=10, N_{2}=16$, and $N_{3}=6$.
} 
scale $M$ are

$$
\begin{aligned}
M_{1} & =\frac{6}{5} N_{1} m_{\mathrm{GM}}, \\
M_{2} & =N_{2} m_{\mathrm{GM}}, \\
M_{3} & =N_{3} m_{\mathrm{GM}} .
\end{aligned}
$$

The two-loop scalar soft masses are

$$
\begin{aligned}
m_{Q}^{2} & =\left(\frac{8}{3} N_{3}+\frac{3}{2} N_{2}+\frac{1}{25} N_{1}\right) m_{\mathrm{GM}}^{2}, \\
m_{U}^{2} & =\left(\frac{8}{3} N_{3}+\frac{16}{25} N_{1}\right) m_{\mathrm{GM}}^{2}, \\
m_{D}^{2} & =\left(\frac{8}{3} N_{3}+\frac{4}{25} N_{1}\right) m_{\mathrm{GM}}^{2}, \\
m_{E}^{2} & =\left(\frac{36}{25} N_{1}\right) m_{\mathrm{GM}}^{2}, \\
m_{H_{u, d}}^{2}=m_{L}^{2} & =\left(\frac{3}{2} N_{2}+\frac{9}{25} N_{1}\right) m_{\mathrm{GM}}^{2} .
\end{aligned}
$$

Here we have assumed that the running between $M_{\mathrm{GUT}}$ and $M$ can be neglected. For $m_{\mathrm{GM}}$ to be $\mathcal{O}\left(10^{2-3}\right) \mathrm{GeV}$, we need $F \simeq\left(\text { few } \times 10^{10} \mathrm{GeV}\right)^{2}$. This implies that gravity mediation is not negligible,

$$
\frac{F}{\sqrt{3} M_{\mathrm{Pl}}}=m_{3 / 2} \simeq m_{\mathrm{GM}} .
$$

However, gaugino and scalar soft masses will be dominated by the gauge-mediated contributions if the $N_{i}$ are moderately large. The $A, B_{\mu}$ and $\mu$ parameters, which are small or absent in minimal gauge mediation, are induced by gravity mediation and will be of the order $m_{3 / 2}$. Unlike in low-scale gauge mediation models, the flavour problem is not automatically solved; we therefore require the gravity-mediated soft terms to be approximately flavour preserving. Incidentally, the particle spectrum in our models will be rather heavy, which of course also helps with evading flavour constraints.

\section{A focus point from high-scale gauge mediation}

Recall that at large $\tan \beta$, the $Z$ mass is given $b^{2}$

$$
-\frac{\widehat{m}_{Z}^{2}}{2}=\left.\left(|\mu|^{2}+m_{H_{u}}^{2}\right)\right|_{m_{\mathrm{S}}}
$$

where $\mu$ and $m_{H_{u}}$ are the running masses at the scale $m_{\mathrm{S}}$ where the Higgs potential is minimized (as usual taken to be $m_{\mathrm{S}}=\sqrt{m_{\tilde{t}_{1}} m_{\tilde{t}_{2}}}$ ). Unlike the Higgsino mass $\mu$, the Higgs soft mass $m_{H_{u}}^{2}$ receives large radiative corrections through its $\mathrm{RG}$ evolution. If the gaugino and scalar soft terms are $\mathcal{O}($ few $\mathrm{TeV})$ while $\mu$ is $\mathcal{O}(100 \mathrm{GeV})$, then the UV-scale value

\footnotetext{
${ }^{2}$ Here and in the following, we use the symbol $\widehat{m}_{Z}$ for the quantity calculated from the MSSM Higgs potential, reserving $m_{Z}$ for the actual physical pole mass $m_{Z}=91.2 \mathrm{GeV}$.
} 
of $m_{H_{u}}^{2}$ must approximately cancel against these radiative corrections for $m_{H_{u}}^{2}$ to end up small (and negative) at $m_{\mathrm{S}}$. We can estimate $\widehat{m}_{Z}$ in terms of the UV-scale parameters as

$$
\begin{aligned}
\widehat{m}_{Z}^{2}= & \left(2.25 M_{3}^{2}-0.45 M_{2}^{2}-0.01 M_{1}^{2}+0.19 M_{2} M_{3}+0.03 M_{1} M_{3}\right. \\
& +0.74 m_{U}^{2}+0.65 m_{Q}^{2}-0.04 m_{D}^{2}-1.32 m_{H_{u}}^{2}-0.09 m_{H_{d}}^{2} \\
& +0.19 A_{0}^{2}-0.40 A_{0} M_{3}-0.11 A_{0} M_{2}-0.02 A_{0} M_{1} \\
& \left.-1.42|\mu|^{2}\right)\left.\right|_{M} .
\end{aligned}
$$

Here the functional form of the r.h.s. follows from the form of the RG equations and from dimensional analysis. The coefficients have been determined assuming a universal trilinear parameter $A_{0}$, and with $M=10^{16} \mathrm{GeV}, m_{\mathrm{S}}=3.5 \mathrm{TeV}, g^{2}(M)=0.484, y_{t}(M)=0.55$, $y_{b}(M)=0.4$, and $y_{\tau}(M)=0.5$; these are typical values for a heavy spectrum at $\tan \beta \approx 50$. We have used two-loop RG equations for the gauge and Yukawa couplings and for the gaugino masses, and one-loop RG equations otherwise. Terms with coefficients smaller than 0.01 have been omitted.

From eq. (3.2) one can immediately read off that, as is well known, the electroweak scale is most sensitive to the gluino mass among all soft parameters. The second line also illustrates the original focus point scenario [3-5]: If $M_{1,2,3}$ and $\mu$ are small, and if there is a universal GUT-scale scalar soft mass $m_{0}$, then $m_{0}$ can be rather large without excessive fine-tuning. Pictorially speaking, the RG trajectories of $m_{H_{u}}^{2}$ for various choices of $m_{0}$ "focus" to always cross zero close to the Fermi scale.

In our class of models, the gaugino masses are by no means small. Any focus pointlike cancellation will have to involve all of the dominant gauge-mediated terms, at least including $M_{3}, M_{2}, m_{H_{u}}^{2}, m_{U}^{2}$ and $m_{Q}^{2}$. Neglecting gravity-mediated contributions for the moment, in particular $\mu$ and $A_{0}$, we can insert eqs. (2.3) and (2.4) into eq. (3.2) to find the contribution to $\widehat{m}_{Z}^{2}$ which is purely due to the gauge-mediated soft terms:

$$
\begin{aligned}
\Delta_{\widehat{m}_{Z}^{2}}^{\mathrm{GM}}= & \left(2.25 N_{3}^{2}-0.45 N_{2}^{2}-0.01 N_{1}^{2}\right. \\
& +0.19 N_{2} N_{3}+0.04 N_{1} N_{3} \\
& \left.+3.80 N_{3}-1.16 N_{2}-0.01 N_{1}\right) m_{\mathrm{GM}}^{2}
\end{aligned}
$$

A small contribution to $\widehat{m}_{Z}^{2}$ results for certain favourable ratios of $N_{2}$ and $N_{3}$, while the influence of $N_{1}$ is subdominant. Indeed there is not even a reason for $N_{1}$ to be integral, since a generic model may contain several hypercharged messengers with various rational hypercharges. Thus, setting $N_{1}=N_{2}$ for simplicity, we find that $\Delta_{\widehat{m}_{Z}^{2}}^{\mathrm{GM}}$ is exceptionally small for

$$
N_{2}=23, \quad N_{3}=9
$$

or

$$
N_{2}=28, \quad N_{3}=11 .
$$

More precisely, for $N_{1}=N_{2}=23$ and $N_{3}=9$ we get a contribution to $\widehat{m}_{Z}^{2}$ which is of the order of $m_{\mathrm{GM}}$, while the actual soft masses are larger by an order of magnitude:

$$
\Delta_{\widehat{m}_{Z}^{2}}^{\mathrm{GM}}=0.6 m_{\mathrm{GM}}^{2}
$$




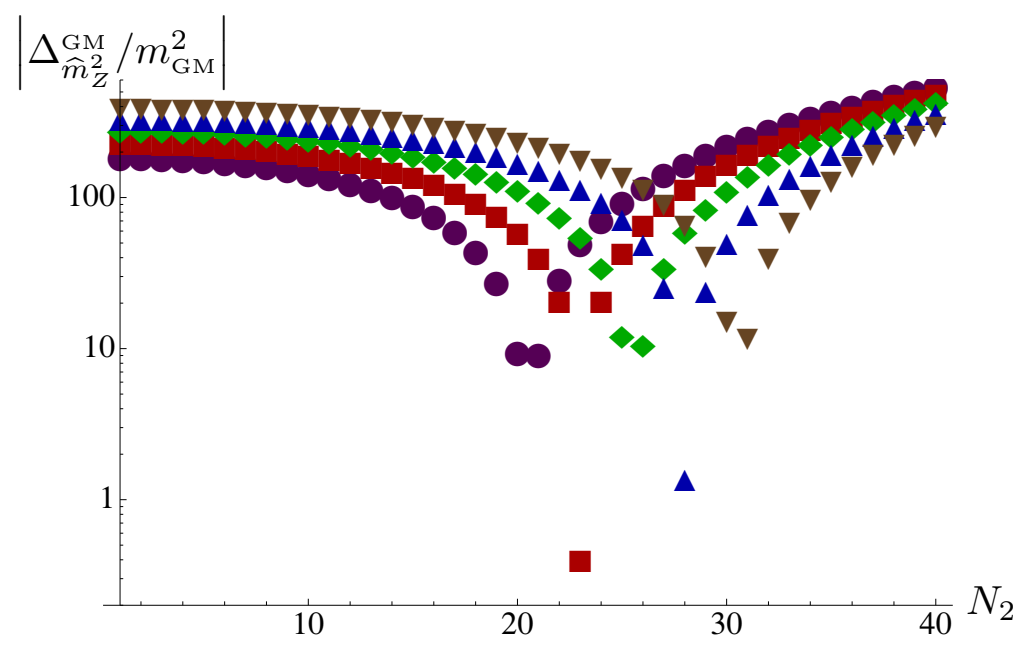

Figure 1. The ratio $\Delta_{\widehat{m}_{Z}^{2}}^{\mathrm{GM}} / m_{\mathrm{GM}}^{2}$ for varying $N_{2}$, with $N_{1}=N_{2}$ and $N_{3}=8$ (purple circles), $N_{3}=9$ (red squares), $N_{3}=10$ (green diamonds), $N_{3}=11$ (blue triangles) and $N_{3}=12$ (brown inverted triangles). On the left branch of each curve, electroweak symmetry is broken, while it is unbroken on the right branch. Note the exceptionally small ratios at $\left(N_{2}, N_{3}\right)=(23,9)$ and $(28,11)$.

Similarly, for $N_{1}=N_{2}=28$ and $N_{3}=11$ one obtains

$$
\Delta_{\widetilde{m}_{Z}^{2}}^{\mathrm{GM}}=1.3 m_{\mathrm{GM}}^{2}
$$

The cancellation in the gauge-mediated contribution to $\widehat{m}_{Z}^{2}$ is illustrated in figure 1 , where we have logarithmically plotted $\Delta_{\widehat{m}_{Z}^{2}}^{\mathrm{GM}}$ in units of $m_{\mathrm{GM}}^{2}$ for various choices of $N_{3}$ and $N_{2}$, after setting $N_{1}=N_{2}$. For positive $\widehat{m}_{Z}^{2}$ (towards smaller $N_{2}$ ) the electroweak symmetry is broken and the magnitude of $\widehat{m}_{Z}^{2}$ represents the electroweak scale. If $\widehat{m}_{Z}^{2}$ is negative (towards larger $N_{2}$ ), the electroweak symmetry is unbroken and its magnitude corresponds to the running Higgs mass squared at the TeV scale. The plot illustrates the well-known problem of quadratic divergences [17]: Generically, the scale of symmetry breaking is given by the cutoff of the theory, in our case the scale of grand unification. In supersymmetric theories, the cutoff is replaced by the supersymmetry breaking mass terms, which reduces the "hierarchy problem" to the "little hierarchy problem". Hence the Higgs mass squared at a $\mathrm{TeV}$ is typically of the order $N_{2,3}^{2} m_{\mathrm{GM}}^{2}$. However, for certain relations between the superparticle mass terms, corresponding to particular messenger indices, cancellations occur and the scale of electroweak symmetry breaking can be of the order $m_{\mathrm{GM}}^{2}$.

In general the soft terms will also receive contributions from gravity mediation. This is especially problematic for $M_{3}$, since changing the gaugino mass ratios resulting from eq. (3.4) or eq. (3.5) by more than a few percent will immediately spoil the cancellation between the soft terms in eq. (3.2), in view of the large coefficient of $M_{3}^{2}$. The dangerous leading gravity-mediated contribution to gaugino masses comes from the operator

$$
\mathcal{L} \supset \int d^{2} \theta \frac{X}{M_{\mathrm{Pl}}} \operatorname{tr} W^{\alpha} W_{\alpha}+\text { h.c. }
$$


Likewise, the $A_{0} M_{i}$ terms in eq. (3.2) are $N_{i}$-enhanced and therefore potentially dangerous. The leading operators inducing $A$-terms are

$$
\begin{aligned}
\mathcal{L} & \supset \int d^{2} \theta \frac{X}{M_{\mathrm{Pl}}}\left(H_{u} Q U+H_{d} Q D+H_{d} L E\right)+\text { h.c. } \\
& +\int d^{4} \theta \frac{X}{M_{\mathrm{Pl}}}\left(\left|H_{u}\right|^{2}+\left|H_{d}\right|^{2}+|Q|^{2}+|U|^{2}+|D|^{2}+|L|^{2}+|E|^{2}\right)+\text { h.c. }
\end{aligned}
$$

All operators of eqs. (3.8) and (3.9) can be forbidden by a symmetry under which $X$ is charged (and which is spontaneously broken by the VEV of $X$ ). By forbidding gravitymediated $A$-terms, we are also removing a major source of dangerous flavour-changing neutral currents. The Giudice-Masiero term

$$
\mathcal{L} \supset \int d^{4} \theta \frac{X^{\dagger}}{M_{\mathrm{Pl}}} H_{u} H_{d}+\text { h.c. }
$$

is forbidden as well, but an effective $\mu$ parameter of the order $m_{3 / 2}$ can still arise from superpotential couplings after SUSY breaking. More precisely, in a SUSY-breaking Minkowski vacuum, the $\mathrm{VEV}$ of the superpotential is of the order $W_{0} \simeq F M_{\mathrm{Pl}}$. A continuous or discrete $R$-symmetry forbids a bare $\mu$ term while allowing for an operator

$$
\mathcal{L} \supset \int d^{2} \theta \frac{W_{0}}{M_{\mathrm{Pl}}^{2}} H_{u} H_{d}+\text { h.c. }
$$

which generates an effective $\mu \simeq F / M_{\mathrm{Pl}}$ [18-20]. Note that the operator

$$
\mathcal{L} \supset \int d^{4} \theta \frac{X^{\dagger} X}{M_{\mathrm{Pl}}^{2}} H_{u} H_{d}+\text { h.c. }
$$

which induces $B_{\mu}$ is allowed in any case, as are the operators for gravity-mediated contributions to scalar soft masses

$$
\mathcal{L} \supset \int d^{4} \theta \frac{X^{\dagger} X}{M_{\mathrm{Pl}}^{2}}\left(\left|H_{u}\right|^{2}+\left|H_{d}\right|^{2}+|Q|^{2}+|U|^{2}+|D|^{2}+|L|^{2}+|E|^{2}\right) .
$$

The latter will give small corrections to the soft masses of eqs. (2.4), without much affecting the focus point cancellation.

The gaugino masses also receive corrections at two-loop and higher orders. However, it was shown in [21] that for large messenger masses and multiplicities, these corrections tend to be very small.

Without radiative corrections, the lightest Higgs mass would be bounded from above by $m_{Z}$, with the bound saturated at large $\tan \beta$. Including the one-loop corrections from the top-stop sector, at large $\tan \beta$ we have (see e.g. [22])

$$
\frac{m_{Z}^{2}}{m_{h_{0}}^{2}}=\left[1+\frac{3}{2 \pi^{2}} \frac{y_{t}^{4}}{g_{1}^{2}+g_{2}^{2}}\left(\log \frac{m_{\mathrm{S}}^{2}}{m_{t}^{2}}+\frac{A_{t}^{2}}{m_{\mathrm{S}}^{2}}\left(1-\frac{A_{t}^{2}}{12 m_{\mathrm{S}}^{2}}\right)\right)\right]^{-1},
$$

where as before $m_{\mathrm{S}}^{2}=m_{\tilde{t}_{1}} m_{\tilde{t}_{2}}$. Note that a Higgs mass around $125 \mathrm{GeV}$ requires radiative corrections of the same size as the tree-level mass. 


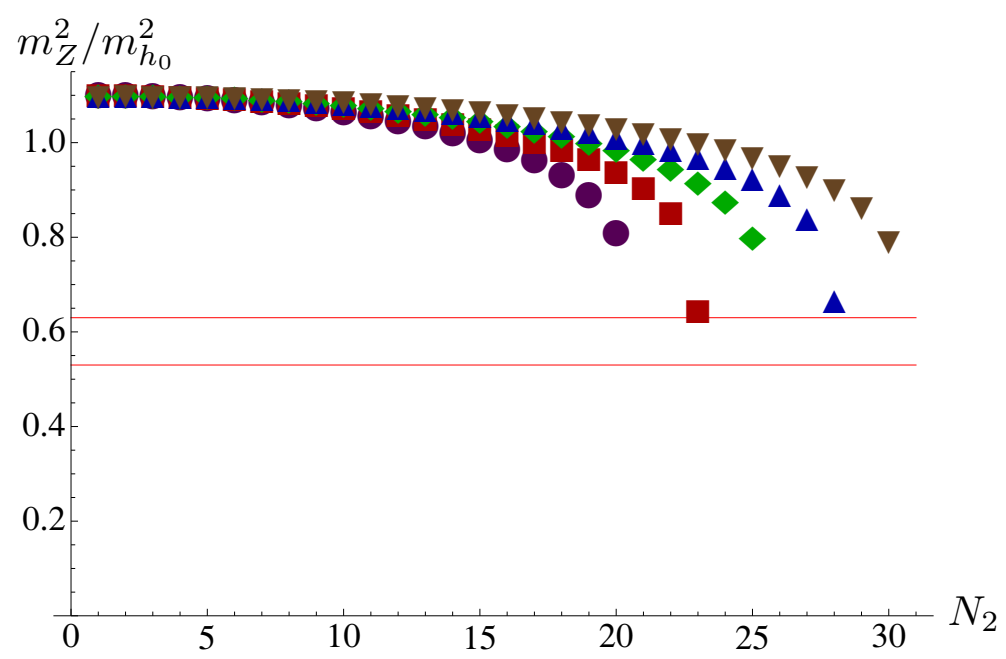

Figure 2. The ratio $m_{Z}^{2} / m_{h_{0}}^{2}$, estimated from eq. (3.14) with gauge-mediated contributions only, for various $N_{2}$ and $N_{3}$ as in figure 1 . The red lines correspond to $m_{h_{0}}=115 \mathrm{GeV}$ (upper line) and $m_{h_{0}}=125 \mathrm{GeV}$ (lower line). While the actual Higgs masses (as computed by SOFTSUSY or FeynHiggs) tend to be even a few GeV higher, such that the $124-126 \mathrm{GeV}$ region can be reached, the figure still shows that for the messenger indices of eqs. (3.4) and (3.5), the enhancement of $m_{h_{0}}$ above $m_{Z}$ is particularly strong.

Exceptionally large radiative corrections to the Higgs mass point to special properties of the Higgs-top-stop system, in our case stop masses much larger than the scale of electroweak symmetry breaking. As discussed above, this situation can be realized if the gauge-mediated contributions to squark masses are large and the electroweak scale emerges as a focus point. As we did for $\widehat{m}_{Z}^{2}$ in eq. (3.2), we can now again express all dimensionful parameters on the r.h.s. of eq. (3.14) as functions of the GUT-scale soft terms, with the coefficients determined from the RG equations (for instance, setting $m_{t}^{2}=\frac{2 y_{t}^{2}}{g_{1}^{2}+g_{2}^{2}} \widehat{m}_{Z}^{2}$ with $\widehat{m}_{Z}^{2}$ taken from eq. (3.2)). The resulting ratio $m_{Z}^{2} / m_{h_{0}}^{2}$ is plotted in figure 2 for various messenger numbers, again using only the gauge-mediated contributions as given by eqs. (2.3) and (2.4). For generic messenger indices, $m_{h_{0}}^{2}$ exceeds $m_{Z}^{2}$ by less than $20 \%$. On the contrary, for messenger indices yielding the Fermi scale as a focus point, $m_{h_{0}}^{2}$ exceeds $m_{Z}^{2}$ by more than $70 \%$.

\section{Phenomenology}

Let us first consider a model where the messenger numbers are given by $N_{1}=N_{2}=23$, $N_{3}=9$. We set $m_{\mathrm{GM}}=200 \mathrm{GeV}$, which corresponds to $F=\left(2.5 \cdot 10^{10} \mathrm{GeV}\right)^{2}$ and is of the same order as $m_{3 / 2} \approx 150 \mathrm{GeV}$ by construction, and as $m_{Z}$. With this data, and with $\mu \simeq \sqrt{B_{\mu}} \simeq m_{3 / 2}$ suitably chosen, we can compute the low-scale particle spectrum at twoloop precision using SOFTSUSY [23]. The lightest Higgs mass is found to be $123.4 \mathrm{GeV}$ by SOFTSUSY and $122.4 \mathrm{GeV}$ by FeynHiggs [24-27]. Taking into account a theoretical uncertainty of at least $1-2 \mathrm{GeV}$, this is marginally compatible with the region currently favoured by ATLAS and CMS. Other features of the spectrum at the electroweak scale include 
- large $\tan \beta \approx 50$, as a consequence of the fact that $B_{\mu}$ is generated by gravity mediation but $m_{H_{u, d}}^{2}$ are dominated by gauge mediation;

- a very heavy gluino, $M_{3} \approx 3.8 \mathrm{TeV}$;

- very heavy squarks, the lightest of which is the $\tilde{t}_{1}$ at about $2.5 \mathrm{TeV}$, while the firstgeneration squarks are all heavier than $3 \mathrm{TeV}$;

- the remaining Higgs bosons $H^{ \pm}, H^{0}$ and $A$ at intermediate masses, at about $1.5 \mathrm{TeV}$ in our benchmark point;

- a right-handed stau as the lightest scalar superparticle;

- three light higgsinos whose mass scale is set by the gravity-mediated $\mu$ parameter. As explained in some detail in [11], these are two neutralinos and a chargino which are nearly degenerate in mass. Their most natural mass range is about $150-300 \mathrm{GeV}$, since the gravitino and the higgsinos should have comparable masses, with the gravitino being the LSP. For a detailed discussion of gravitino dark matter and the BBN problem in these models, see again [11].

A similar but even slightly heavier spectrum is obtained with $N_{1}=N_{2}=28, N_{3}=$ 11. Spectra with $N_{1}<N_{2}$ can also be found. Interestingly, upon decreasing $N_{1}$ the $\tilde{\tau}_{1}$ can become rather light, as a consequence of large $\tan \beta$ and the fact that the righthanded stau soft mass is induced only by hypercharged messengers. For comparison, we have summarized the masses of three benchmark spectra in table 1: the above one with $\left(N_{1}, N_{2}, N_{3}\right)=(23,23,9)$; one with $(17,23,9)$ and a relatively light $\tilde{\tau}_{1}$; and one with $(28,28,11)$.

Admittedly, the choice of parameters described above is probably at the boundary of what might be considered acceptable. Large messenger multiplicities will lead to very large threshold corrections to gauge coupling unification, and the theoretical uncertainties in the calculation of the spectrum are considerable for multi-TeV soft masses. In addition, the Higgs mass is at the lower end of the range indicated by experiment.

A spectrum like this comes close to a "nightmare scenario" for the LHC, since all coloured particles are kinematically out of reach. The light higgsinos will be produced in electroweak processes, but their decay signals are extremely difficult to distinguish from Standard Model backgrounds [28, 29]. It may be possible to see a signal in monojet searches, where a higgsino pair is produced in the Drell-Yan process and shows up as missing $E_{T}$, while a gluon is radiated from the initial state. The $\tilde{\tau}_{1}$ could also be within LHC reach if $N_{1}$ happens to be sufficiently small, but will be similarly difficult to detect. The situation will be much better at a linear collider, where all three higgsinos and possibly the $\tilde{\tau}_{1}$ can be produced and their masses and couplings can be accurately measured.

\section{$5 \quad$ Fine-tuning}

One might object to our approach on the grounds that the coefficients in eq. (3.2) are disturbingly sensitive to variations in the Yukawa couplings, the gauge couplings, and to 


\begin{tabular}{|cccc|}
\hline particle & $(23,23,9)$ model & $(17,23,9)$ model & $(28,28,11)$ model \\
\hline$h_{0}$ & 123 & 123 & 124 \\
$\chi_{1}^{0}$ & 205 & 205 & 164 \\
$\chi_{1}^{ \pm}$ & 207 & 206 & 166 \\
$\chi_{2}^{0}$ & 208 & 207 & 167 \\
\hline$\tilde{\tau}_{1}$ & 1530 & 550 & 1890 \\
$H^{0}$ & 1470 & 1110 & 2200 \\
$A$ & 1480 & 1120 & 2200 \\
$H^{ \pm}$ & 1480 & 1120 & 2200 \\
\hline$\chi_{3}^{0}$ & 2500 & 1800 & 2700 \\
$\chi_{4}^{0}$ & 3800 & 3800 & 4100 \\
$\chi_{2}^{ \pm}$ & 3800 & 3800 & 4100 \\
\hline$\tilde{g}$ & 3800 & 3800 & 4200 \\
$\tilde{t}_{1}$ & 2500 & 2300 & 2700 \\
$\tilde{u}_{1}$ & 3700 & 3500 & 4000 \\
$\tilde{d}_{1}$ & 3400 & 3400 & 3700 \\
\hline
\end{tabular}

Table 1. Some selected masses in GeV, computed with SOFTSUSY, for three models with messenger indices $\left(N_{1}, N_{2}, N_{3}\right)=(23,23,9),(17,23,9)$, and $(28,28,11)$. The first has $m_{\mathrm{GM}}=200 \mathrm{GeV}$, $\mu=240 \mathrm{GeV}$, and $\tan \beta=50$; the second, $m_{\mathrm{GM}}=200 \mathrm{GeV}, \mu=250 \mathrm{GeV}$, and $\tan \beta=52$; and the third, $m_{\mathrm{GM}}=180 \mathrm{GeV}, \mu=180 \mathrm{GeV}$, and $\tan \beta=44$.

the overall scale of SUSY breaking. The worst sensitivity is with respect to the latter two: For example, changing the value of $g^{2}(M)$ from 0.484 to 0.5 while keeping all other parameters fixed, eq. (3.2) becomes

$$
\widehat{m}_{Z}^{2}=\left.\left(2.50 M_{3}^{2}-0.45 M_{2}^{2}-0.01 M_{1}^{2}+\ldots\right)\right|_{M}
$$

and after inserting the messenger numbers $\left(N_{1}, N_{2}, N_{3}\right)=(23,23,9)$, the cancellation in eq. (3.6) is lost:

$$
\Delta_{\widehat{m}_{Z}^{2}}^{\mathrm{GM}}=26 m_{\mathrm{GM}}^{2} .
$$

Similarly, keeping $g^{2}(M)=0.483$ but changing $m_{\mathrm{S}}$ to $10 \mathrm{TeV}$ (which is of course equivalent to an appropriate change of the background field $F$ ) gives

$$
\widehat{m}_{Z}^{2}=\left.\left(1.98 M_{3}^{2}-0.45 M_{2}^{2}-0.01 M_{1}^{2}+\ldots\right)\right|_{M}
$$

and eq. (3.6) becomes

$$
\Delta_{\widehat{m}_{Z}^{2}}^{\mathrm{GM}}=-31 m_{\mathrm{GM}}^{2} .
$$

Is our focus point therefore merely relegating the fine-tuning to these parameters? Or, to put it more drastically, would we still consider this scenario natural in the hypothetical situation that the Standard Model gauge couplings and the EWSB scale had not yet been measured? 
While we cannot dispel these objections altogether, they might at least be mitigated by the following arguments. In the String Landscape we expect all fundamental parameters to be discrete, and the fine-tuning problem to disappear. The question is if we can realize a mechanism by which this happens already in effective field theory. At present it seems impossible to identify, within this framework, a physical principle which fixes the GUTscale gauge coupling to exactly its measured value, without introducing additional free continuous parameters. The same is true for the SUSY breaking scale. However, in realistic string models these two quantities are of course correlated. In fact, in the heterotic models by which our models are inspired, they are correlated in a rather precisely defined way: The gauge coupling is set by the vacuum expectation value of the dilaton $S$,

$$
\frac{8 \pi^{2}}{g^{2}}=S
$$

while the scale of dynamical SUSY breaking is set by the strong-coupling scale $\Lambda$ of some non-abelian gauge group in the hidden sector, which is ultimately related to the dilaton by

$$
\sqrt{F} \simeq \Lambda \simeq e^{-S / b_{0}} .
$$

Decreasing $S$ will simultaneously increase $g(M)$ and $m_{\mathrm{S}}$, and the resulting effects on $\Delta_{\widehat{m}_{Z}^{2}}^{\mathrm{GM}}$ can cancel (as should be clear from the above example) for a suitable value of the discrete quantity $b_{0}$. Of course the actual value of $b_{0}$ is model-dependent; nevertheless, this shows that it may not be sensible to treat the gauge coupling and the SUSY breaking scale as independent parameters.

On the other hand, changing the continuous parameters slightly can give new characteristic relations between the messenger indices which again lead to a focus point at the electroweak scale. Empirically, we find that these always occur at a $N_{3}: N_{2}$ ratio of around 0.39 (e.g. for our models we have $11 / 28 \approx 0.393$ and $9 / 23 \approx 0.391$ ). To determine which of them best describes our universe, it would however be necessary to measure the mass ratios of the supersymmetric particles rather precisely — a task which is clearly beyond the capabilities of the LHC for multi-TeV soft masses, but does not seem impossible in principle.

\section{Conclusions}

In this note we have argued that the little hierarchy between the EWSB scale and the MSSM soft masses may be due to a focus point exhibited by models of high-scale gauge mediation. Since they are determined by discrete messenger indices, the soft terms can be of the order of several $\mathrm{TeV}$ without large fine-tuning. We have identified two favourable ratios of messenger indices for $\mathrm{SU}(2)$ and $\mathrm{SU}(3)$ fundamentals, $\left(N_{2}, N_{3}\right)=(23,9)$ and $(28,11)$. With these rather large multiplicities, the spectrum can be made compatible with the recent experimental evidence for a $124-126 \mathrm{GeV}$ Higgs. Unfortunately, for this choice of parameters almost all superpartners are beyond the reach of the LHC. The only supersymmetric particles with weak-scale masses are the higgsinos and possibly the lightest stau, all of which would probably need a linear collider to be discovered, and the gravitino LSP. 


\section{Acknowledgments}

The authors thank G. Ross for helpful discussions.

Open Access. This article is distributed under the terms of the Creative Commons Attribution License which permits any use, distribution and reproduction in any medium, provided the original author(s) and source are credited.

\section{References}

[1] ATLAS collaboration, Combination of Higgs Boson Searches with up to $4.9 \mathrm{fb}^{-1}$ of $\mathrm{pp}$ Collisions Data Taken at a center-of-mass energy of $7 \mathrm{TeV}$ with the ATLAS Experiment at the LHC, ATLAS-CONF-2011-163 (2011).

[2] CMS collaboration, Combination of SM Higgs Searches, CMS-PAS-HIG-11-032 (2011).

[3] K.L. Chan, U. Chattopadhyay and P. Nath, Naturalness, weak scale supersymmetry and the prospect for the observation of supersymmetry at the Tevatron and at the CERN LHC, Phys. Rev. D 58 (1998) 096004 [hep-ph/9710473] [INSPIRE].

[4] J.L. Feng, K.T. Matchev and T. Moroi, Multi-TeV scalars are natural in minimal supergravity, Phys. Rev. Lett. 84 (2000) 2322 [hep-ph/9908309] [INSPIRE].

[5] J.L. Feng, K.T. Matchev and T. Moroi, Focus points and naturalness in supersymmetry, Phys. Rev. D 61 (2000) 075005 [hep-ph/9909334] [INSPIRE].

[6] S. Akula, M. Liu, P. Nath and G. Peim, Naturalness, supersymmetry and implications for LHC and dark matter, Phys. Lett. B 709 (2012) 192 [arXiv:1111.4589] [INSPIRE].

[7] J.L. Feng, K.T. Matchev and D. Sanford, Focus point supersymmetry redux, Phys. Rev. D 85 (2012) 075007 [arXiv: 1112.3021] [INSPIRE].

[8] H. Abe, T. Kobayashi and Y. Omura, Relaxed fine-tuning in models with non-universal gaugino masses, Phys. Rev. D 76 (2007) 015002 [hep-ph/0703044] [INSPIRE].

[9] D. Horton and G. Ross, Naturalness and focus points with non-universal gaugino masses, Nucl. Phys. B 830 (2010) 221 [arXiv:0908.0857] [INSPIRE].

[10] J.E. Younkin and S.P. Martin, Non-universal gaugino masses, the supersymmetric little hierarchy problem and dark matter, Phys. Rev. D 85 (2012) 055028 [arXiv:1201.2989] [INSPIRE].

[11] F. Brümmer and W. Buchmüller, Light Higgsinos as heralds of higher-dimensional unification, JHEP 07 (2011) 010 [arXiv:1105.0802] [INSPIRE].

[12] S. Heinemeyer, O. Stal and G. Weiglein, Interpreting the LHC Higgs search results in the MSSM, Phys. Lett. B 710 (2012) 201 [arXiv:1112.3026] [InSPIRE].

[13] A. Arbey, M. Battaglia, A. Djouadi, F. Mahmoudi and J. Quevillon, Implications of a 125 GeV Higgs for supersymmetric models, Phys. Lett. B 708 (2012) 162 [arXiv:1112.3028] [INSPIRE].

[14] P. Draper, P. Meade, M. Reece and D. Shih, Implications of a $125 \mathrm{GeV}$ Higgs for the MSSM and low-scale SUSY breaking, arXiv:1112.3068 [INSPIRE].

[15] M. Carena, S. Gori, N.R. Shah and C.E. Wagner, A $125 \mathrm{GeV}$ SM-like Higgs in the MSSM and the $\gamma \gamma$ rate, JHEP 03 (2012) 014 [arXiv:1112.3336] [INSPIRE]. 
[16] S. Akula, B. Altunkaynak, D. Feldman, P. Nath and G. Peim, Higgs boson mass predictions in SUGRA unification, recent LHC-7 results and dark matter, Phys. Rev. D 85 (2012) 075001 [arXiv: 1112.3645] [INSPIRE].

[17] M. Veltman, The infrared-ultraviolet connection, Acta Phys. Polon. B 12 (1981) 437 [INSPIRE].

[18] J. Casas and C. Muñoz, A natural solution to the mu problem, Phys. Lett. B 306 (1993) 288 [hep-ph/9302227] [INSPIRE].

[19] W. Buchmüller and J. Schmidt, Higgs versus matter in the heterotic landscape, Nucl. Phys. B 807 (2009) 265 [arXiv:0807.1046] [INSPIRE].

[20] F. Brümmer, R. Kappl, M. Ratz and K. Schmidt-Hoberg, Approximate R-symmetries and the mu term, JHEP 04 (2010) 006 [arXiv: 1003.0084] [INSPIRE].

[21] N. Arkani-Hamed, G.F. Giudice, M.A. Luty and R. Rattazzi, Supersymmetry breaking loops from analytic continuation into superspace, Phys. Rev. D 58 (1998) 115005 [hep-ph/9803290] [INSPIRE].

[22] S.P. Martin, A supersymmetry primer, hep-ph/9709356 [INSPIRE].

[23] B. Allanach, SOFTSUSY: a program for calculating supersymmetric spectra, Comput. Phys. Commun. 143 (2002) 305 [hep-ph/0104145] [INSPIRE].

[24] M. Frank, T. Hahn, S. Heinemeyer, W. Hollik, H. Rzehak and G. Weiglein, The Higgs boson masses and mixings of the complex MSSM in the Feynman-diagrammatic approach, JHEP 02 (2007) 047 [hep-ph/0611326] [INSPIRE].

[25] G. Degrassi, S. Heinemeyer, W. Hollik, P. Slavich and G. Weiglein, Towards high precision predictions for the MSSM Higgs sector, Eur. Phys. J. C 28 (2003) 133 [hep-ph/0212020] [INSPIRE].

[26] S. Heinemeyer, W. Hollik and G. Weiglein, The masses of the neutral CP-even Higgs bosons in the MSSM: accurate analysis at the two loop level, Eur. Phys. J. C 9 (1999) 343 [hep-ph/9812472] [INSPIRE].

[27] S. Heinemeyer, W. Hollik and G. Weiglein, FeynHiggs: a program for the calculation of the masses of the neutral CP even Higgs bosons in the MSSM, Comput. Phys. Commun. 124 (2000) 76 [hep-ph/9812320] [INSPIRE].

[28] H. Baer, V. Barger and P. Huang, Hidden SUSY at the LHC: the light higgsino-world scenario and the role of a lepton collider, JHEP 11 (2011) 031 [arXiv:1107.5581] [INSPIRE].

[29] S. Bobrovskyi, F. Brümmer, W. Buchmüller and J. Hajer, Searching for light higgsinos with b-jets and missing leptons, JHEP 01 (2012) 122 [arXiv:1111.6005] [INSPIRE]. 